Case Report

\title{
A Case of Septicemia due to Nonocclusive Mesenteric Ischemia Occurring in Induction Chemotherapy
}

\author{
Hideki Tanaka (D), Kiyoaki Tsukahara, Isaku Okamoto, Rio Kojima, Kazuhiro Hirasawa, \\ and Hiroki Sato
}

Department of Otorhinolaryngology, Head and Neck Surgery, Tokyo Medical University, Tokyo, Japan

Correspondence should be addressed to Hideki Tanaka; hide.cola31@gmail.com

Received 8 December 2017; Revised 18 March 2018; Accepted 25 March 2018; Published 19 April 2018

Academic Editor: Dinesh K. Chhetri

Copyright (c) 2018 Hideki Tanaka et al. This is an open access article distributed under the Creative Commons Attribution License, which permits unrestricted use, distribution, and reproduction in any medium, provided the original work is properly cited.

\begin{abstract}
In nonocclusive mesenteric ischemia (NOMI), mesenteric ischemia and intestinal necrosis occur despite the absence of organic blockage in mesenteric blood vessels. As abdominal pain is often absent and few characteristic findings are seen in blood biochemistry, imaging diagnosis or other examinations, discovery is often delayed. With a mortality rate of 56-79\%, NOMI is a very serious disease. However, few reports have described this pathology in association with chemotherapy regimens such as those used for malignant head and neck tumors. We encountered a case of NOMI during induction therapy combining cisplatin, docetaxel, and 5-fluorouracil. The patient was a 74-year-old man receiving chemotherapy for T2N2bM0 stage IVA oropharyngeal carcinoma. Febrile neutropenia appeared on treatment day 8. An antibacterial agent and a granulocyte colony-stimulating factor were administered, but septic shock developed and he was transferred to the intensive care unit. Abdominal distension was present and contrast-enhanced computed tomography of the abdomen suggested NOMI. Emergency surgery on day 9 resected the necrotized small intestine and created a single-hole ileostomy. The patient subsequently recovered with 2 weeks of continuous hemodiafiltration and other intensive therapies. Otolaryngological surgeons seldom encounter intestinal diseases, which are thus easily overlooked. The present case report may help in achieving early diagnosis.
\end{abstract}

\section{Introduction}

Induction chemotherapy combining the 3 agents of cisplatin (CDDP), docetaxel (DOC), and 5-fluorouracil (5-FU) is standard treatment in head and neck cancer. This tripletherapy is also referred to as TPF (Taxotere, Platinol, and fluorouracil). As TPF is a powerful therapy, adverse events are frequent, and sometimes fatal.

Nonocclusive mesenteric ischemia (NOMI) is an intestinal blood flow disorder without blockage of the main trunk of the mesenteric artery. NOMI has few specific clinical symptoms and findings on medical examinations. As diagnosis is often delayed and irreversible intestinal necrosis results, NOMI is a serious disease. This pathology has a wide range of causes, and chemotherapy has been reported as one. However, no previous reports have described NOMI occurring as a complication of chemotherapy in head and neck cancer [1-4]. We report herein a case of NOMI due to induction chemotherapy with TPF for oropharyngeal carcinoma.

\section{Case Details}

The patient was a 74-year-old man who was examined for the major complaint of swelling in the right neck. His medical history included pneumonia and sensorineural hearing loss, but no family history of note was elicited. He had smoked 40 cigarettes a day until 32 years old. He had first noted swelling of the right neck 5 years earlier, and had been referred to our department in April 2016 after noticing a growing tendency. Pharyngolaryngeal endoscopy had revealed swelling of the lateral wall on the right side of the oropharynx. On cervicothoracic contrast-enhanced computerized tomography (CT), a mass with a maximum diameter of $24 \mathrm{~mm}$ was noted in the lateral wall of the right oropharynx, but no infiltration into the parapharyngeal space or epiglottis was seen. Swelling was also observed in the right upper and middle deep neck lymph nodes and the right node of Rouviere. In blood biochemistry, a squamous cell carcinoma-related antigen was elevated to $2.8 \mathrm{ng} / \mathrm{mL}$. Biopsy of the right oropharynx lateral wall revealed 
p40-and p16-positive squamous cell carcinoma. The diagnosis was T2N2bM0 stage IVA oropharyngeal carcinoma of the right lateral wall. The treatment plan was to manage the disease through induction chemotherapy with TPF. As induction chemotherapy, CDDP and DOC were both administered at $60 \mathrm{mg} / \mathrm{m}^{2}$ on day 1 and 5 -FU at $600 \mathrm{mg} / \mathrm{m}^{2}$ from day 1 to day 5 . Daily urine volume was $\geq 3,000 \mathrm{~mL}$. Although bodyweight decreased day by day from day 4 , compared to before the start of chemotherapy, no deterioration in patient condition had been seen; so, we kept the patient under observation with particular attention to vital signs. On day 8, neutrophil count had dropped to $2250 / \mu \mathrm{L}$ and blood pressure fell to $70 / 50 \mathrm{mmHg}$. However, as no fever was evident, febrile neutropenia was ruled out. As fluid balance continued to be negative each day, we conducted fluid replacement for extracellular fluids to prevent dehydration. The diarrhea that appeared was considered to be drug-induced colitis due to 5FU, and symptomatic treatment was initiated. On day 9 , he showed a fever of $38.0^{\circ} \mathrm{C}$, and neutrophil count had decreased to $212 / \mu \mathrm{L}$. Febrile neutropenia was diagnosed, and administration of cefepime (CFPM), an antibacterial agent, and a granulocyte colony-stimulating factor (G-CSF) agent was commenced. However, $3 \mathrm{~h}$ later, the patient lost consciousness and blood pressure fell to 50/40 $\mathrm{mmHg}$. Septic shock was diagnosed, and he was transferred to the Intensive Care Unit (ICU). Treatment of the septic shock according to early goaldirected therapy was combined with continuous hemodiafiltration and polymyxin B-immobilized fiber column hemoperfusion. As nausea and marked abdominal distension were present, contrast-enhanced CT of the abdomen was conducted (Figures 1 and 2). While a decrease in contrast effect was observed in the small intestine wall, no prominent thrombosis of the mesenteric artery or veins was observed, so NOMI was considered. In consultation with our gastrointestinal surgery department, conservative therapy was considered problematic, and the decision was made to conduct emergency surgery. Partial resection of the small intestine was performed and an ileostomy was created. Numerous ischemic locations were seen in the small intestine, and about $1 \mathrm{~m}$ of the intestine was resected (Figure 3). No intestinal perforation or intraperitoneal contamination was observed. Postoperative pathological examination showed necrosis in the intestinal wall but no organic blockage or blood clots in the mesenteric artery.

On day 2 after surgery, the shock abated. On day 12, the patient was returned to the general ward from the ICU. Contrast-enhanced CT of the neck on postoperative day 15 showed partial response of the oropharyngeal carcinoma. As additional chemotherapy was considered problematic, treatment with cetuximab was conducted in combination with radiotherapy. Both the primary cancer and neck lymph node lesions subsequently disappeared. After half a year, the ileostomy was closed off. In the 9 months following the completion of treatment, no recurrence or metastasis was observed.

\section{Discussion}

NOMI is a disease of the mesenteric blood vessels without organic blockage that causes mesenteric ischemia and

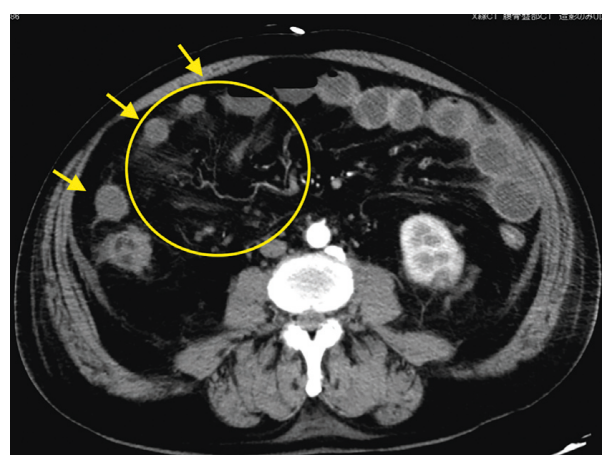

Figure 1: Axial-section contrast-enhanced CT of the abdomen performed in the ICU. Poor contrast effects can be seen in the small intestine (arrows). Disruption of peripheral blood vessels of the superior mesenteric artery is apparent (circled).

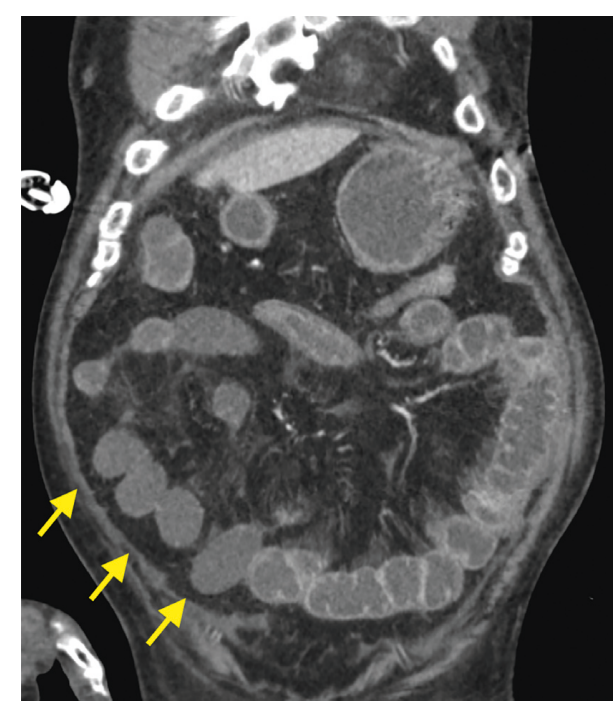

FIgure 2: Coronal-section contrast-enhanced CT of the abdomen performed in the ICU. Poor contrast effects can be seen in the small intestine (arrows).

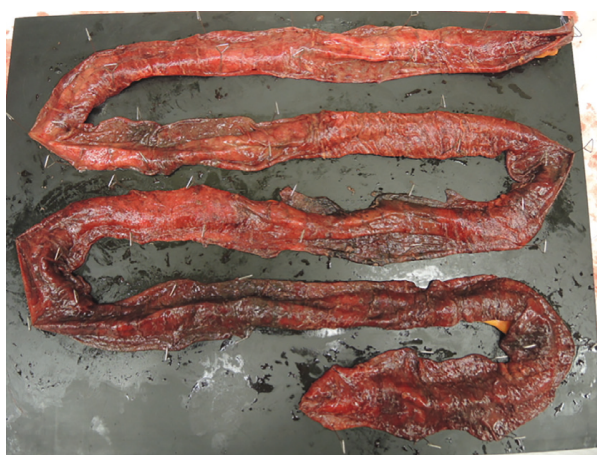

FIgURE 3: Resected small intestine. Multiple ischemic locations are seen, for around $200 \mathrm{~cm}$ from the ligament of Treitz to about $10 \mathrm{~cm}$ from the terminal ileum.

intestinal necrosis. This pathology is the cause of $15-27 \%$ of intestinal ischemia and shows a high mortality rate of $56-79 \%[5,6]$. However, patients frequently show few 
TABLE 1: Previously reported case of NOMI during chemotherapy.

\begin{tabular}{|c|c|c|c|c|}
\hline Author & Age/sex & Cancer region & Chemotherapy regimen & Year reported \\
\hline Pearson et al. [3] & $53 /$ female & Metastatic liver cancer & CDDP, ADM, MMC & 2008 \\
\hline Awano et al. [1] & $80 /$ female & Lung Adenocarcinoma & gefitinib & 2013 \\
\hline Matsuzawa et al. [4] & $74 /$ female & Melanoma & DTIC, ACNU, VCR, $ß$-interferon & 2015 \\
\hline Yamane et al. [2] & 68/male & Small cell lung cancer & CDDP, ETP & 2015 \\
\hline Present case & 74/male & Oropharynx cancer & CDDP, 5-FU, DOC & 2017 \\
\hline
\end{tabular}

CDDP: cisplatin; ADM: adriamycin; MMC: mitomycin C; CBDCA: carboplatin; DTIC: dacarbazine; ACNU: nimustine; VCR: vincristine; ETP: etoposide; DOC: docetaxel.

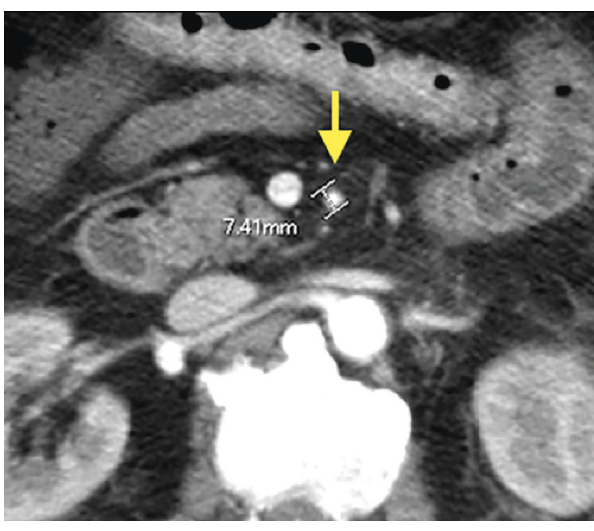

(a)

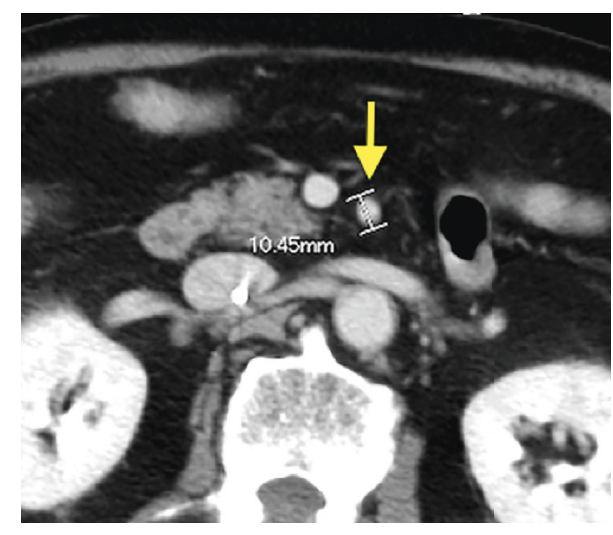

(b)

Figure 4: Coronal-section contrast-enhanced CT of the abdomen. (a) Before surgery. (b) 3 months after surgery. The diameter of the superior mesenteric artery is $7.41 \mathrm{~mm}$ before surgery and $10.45 \mathrm{~mm}$ at 3 months after surgery (arrows).

findings from blood biochemistry or imaging examinations. One reason for the high mortality rate is delayed discovery because of the lack of characteristic findings. Abdominal distension and abdominal pain were observed in the present case, but abdominal pain is not seen in $20-30 \%$ of cases [7-9].

The major features in the pathology of NOMI are an extreme drop and unevenness in intestinal blood flow. Another cause is peripheral vascular spasm due to exaggerated responses in sympathetic nerves and fluid factors, such as vasopressin and angiotensin, accompanying decreases in cardiac output and circulating plasma volume [10]. The three major risks are thus congestive heart failure, digitalis intoxication, and hemagglutination. Other causes include sympathomimetic drugs, diuretics, dialysis, and septic shock. However, chemotherapy has not been reported as a risk factor for NOMI.

A search of PubMed for NOMI and chemotherapy found only 4 cases of NOMI due to chemotherapy (Table 1 ). However, the sites of the primary cancer and regimens varied. Various adverse events occur with chemotherapy and may cause NOMI as a secondary effect. In the present case, daily decreases in bodyweight were seen from day 4 of administering TPF, and hemoconcentration, one of the risk factors for NOMI, occurred. Also, as septic shock resulted from febrile neutropenia, impaired blood flow in the intestinal mucosa was considered to have been exacerbated.

Drug-induced colitis may occur as an adverse event of the 5-FU used in TPF therapy. Symptoms are diarrhea and nausea in mild cases and hemorrhagic colitis and necrotizing enterocolitis in serious cases, resembling NOMI. However, major differences exist between these pathologies regarding treatment and prognosis. In 5-FU-induced colitis, $1.8 \%$ of all diarrhea that occurs may be fatal [11]. Furthermore, NOMI may be hidden among patients diagnosed with 5-FUinduced colitis.

With the NOMI diagnosis algorithm used in the United States, selective angiography has been suggested as the number-one diagnostic modality. However, when NOMI is discovered, systemic conditions are frequently unstable. The rate of conducting selective angiography is thus reportedly low [12]. Diagnostic criteria for mesenteric vascular spasm are proximal stenosis of the superior mesenteric artery and main branch, abnormal images of mesenteric branches, arcade spasm of the mesenteric artery, and poor contrast on internal images of the blood vessels in the mesenteric wall.

The appearance of multidetector row CT (MDCT) in recent years has improved the visualization of veins. MDCT allows angiography diagnostic criteria-related findings to be evaluated [13]. With MDCT, it is also possible to diagnose loss of blood flow contrast effect in the intestinal wall and thinning of the intestinal wall due to decreased mesenteric blood flow. Intestinal ischemia is regional in nature, from the small intestine to the colon, and is distributed unevenly. Regarding the present case, the presence of an extensive poor contrast effect in the intestinal wall and vascular disruption in the superior mesenteric artery periphery on MDCT led to the diagnosis of NOMI. Compared with the results of CT 3 months after surgery, the superior mesenteric artery appeared narrowed on CT before surgery (Figure 4). However, 
angiography should be performed in accordance with the American standards where possible. Regrettably, due to the emergency nature of the intervention and in-hospital circumstances, we were unable to perform angiography.

Thinking that the present case represented 5-FUinduced colitis, the patient had initially been put under observation, with only fluid replenishment and symptomatic treatment. If we had performed MDCT at that time, the disease state may not have become as serious. The drug 5-FU and its derivatives are a major part of treatment in head and neck cancer, and inclusion of NOMI among the differential diagnoses of colitis is therefore important.

Treatment of NOMI can be divided into surgical and nonsurgical methods. For the latter, continuous intraarterial infusion of vasodilators, such as papaverine hydrochloride, is useful. Peritoneal irritation symptoms are often an indicator for surgery. However, in many cases, physical findings cannot be relied upon in elderly patients and those in a poor state of consciousness. Nakao et al. actively performed surgery without angiography for 17 patients with NOMI complicated by shock, and found that colectomy was necessary in 14 patients (82\%) [14].

If an irreversible ischemic state is determined to have developed in the large intestine, as in the present case, surgery is absolutely necessary. In our patient, prompt diagnosis and surgical intervention enabled early infection control, and the early discovery of NOMI potentially saved his life. The emphasis should thus be on early diagnosis of NOMI by means of contrast-enhanced MDCT and teamwork in therapy involving intensive care specialists and gastroenterological surgeons.

\section{Conclusion}

We encountered a rare case of NOMI occurring during induction chemotherapy for head and neck cancer. Otolaryngology department surgeons such as ourselves seldom encounter intestinal diseases and may overlook them. For NOMI in particular, characteristic clinical findings are few, and differentiation from 5-FU-induced colitis is difficult. However, contrast-enhanced MDCT is useful for distinguishing between these pathologies. The unstable hemodynamics in chemotherapy may cause NOMI. The association between chemotherapy and NOMI should be verified in a larger population.

\section{Conflicts of Interest}

The authors have no conflicts of interest to disclose.

\section{References}

[1] N. Awano, K. Kondoh, T. Andoh, S. Ikushima, T. Kumasaka, and T. Takemura, "Three cases of lung carcinoma complicated by gastrointestinal necrosis and perforation," Haigan, vol. 53, no. 6, pp. 809-814, 2013.

[2] H. Yamane, N. Fukuda, K. Nishino et al., "Non-occlusive mesenteric ischemia after splenic metastasectomy for small-cell lung cancer," Internal Medicine, vol. 54, no. 7, pp. 743-747, 2015.
[3] A. C. Pearson, S. Steinberg, M. H. Shah, and M. Bloomston, "The complicated management of patient following transarterial chemoembolization for metastatic carcinoid," World Journal of Surgical Oncology, vol. 6, no. 1, p. 125, 2008.

[4] M. Matsuzawa, K. Harada, N. Hosomura et al., "Nonocclusive mesenteric ischemia after chemotherapy for metastatic melanoma," Journal of Dermatology, vol. 42, no. 1, pp. 105-106, 2015.

[5] Y. Mishima, "Acute mesenteric ischemia," Japanese Journal of Surgery, vol. 18, no. 6, pp. 615-619, 1988.

[6] S. J. Boley, S. Sprayregan, S. S. Siegelman, and F. J. Veith, "Initial results from an aggressiveroentgenologial and surgicall approach to acute mesenteric ischemia," Surgery, vol. 82, pp. 848-855, 1977.

[7] L. G. Britt and R. C. Cheek, "Nonocclusive mesenteric cascular disease: clinical and experimental observantions," Annals of Surgery, vol. 169, no. 5, pp. 704-711, 1969.

[8] S. Klotz, T. Vestring, J. Rotker, C. Schmidt, H. H. Scheld, and C. Schmid, "Diagnosis and treatment of nonocclusive mensenteric ischema after open heart surgery," Annals of Thoracic Surgery, vol. 72, no. 5, pp. 1583-1586, 2001.

[9] M. Trompeter, T. Brazda, C. T. Remy et al., "Non-occlusive mesenteric ischemia: etiology, diagnosis, and intervenetional therapy," European Radiology, vol. 12, no. 5, pp. 1179-1187, 2002.

[10] T. Yamaguchi, "Experimental studies on the etiology of gas in the portal venous system," Japanese Journal of Gastroenterological Surgery, vol. 13, no. 11, pp. 1260-1270, 1980.

[11] H. Ogi, Y. Yoshitake, T. Tanaka, M. Yoneda, M. Maki, and M. Shinohara, "A case of serious drug-induced enteritis that developed during chemoradiotherapy with S-1 in a patient with oral cancer," Japanese Journal of Oral and Maxillofacial Surgery, vol. 62, no. 7, pp. 352-357, 2016.

[12] T. J. Howard, L. A. Plaskon, E. A. Wiebke, M. G. Wilcox, and J. A. Madura, "Nonocclusive mesenteric ischemia remains a diagnostic dilemma," American Journal of Surgery, vol. 171, no. 4, pp. 405-458, 1996.

[13] R. Woodhams, H. Nishimaki, K. Fujii et al., "Usefulness of multidetector-row CT(MDCT) for the diagnosis of nonocclusive mesenteric ischemia (NOMI): assessment of morphology and diameter of the superior mesenteric artery (SMA) on multi-planar reconstructed (MPR) images," European Journal of Radiology, vol. 76, no. 1, pp. 96-102, 2010.

[14] S. Nakao, H. Watanabe, Y. Takahashi, H. Yamamoto, and T. Matsuoka, "Effectiveness of emergency laparotomy for diagnosis and treatment of severe non-occlusive mesenteric ischemia (NOMI)," Journal of Abdominal Emergency Medicine, vol. 31, no. 7, pp. 1021-1027, 2011. 


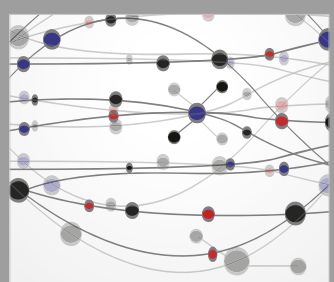

The Scientific World Journal
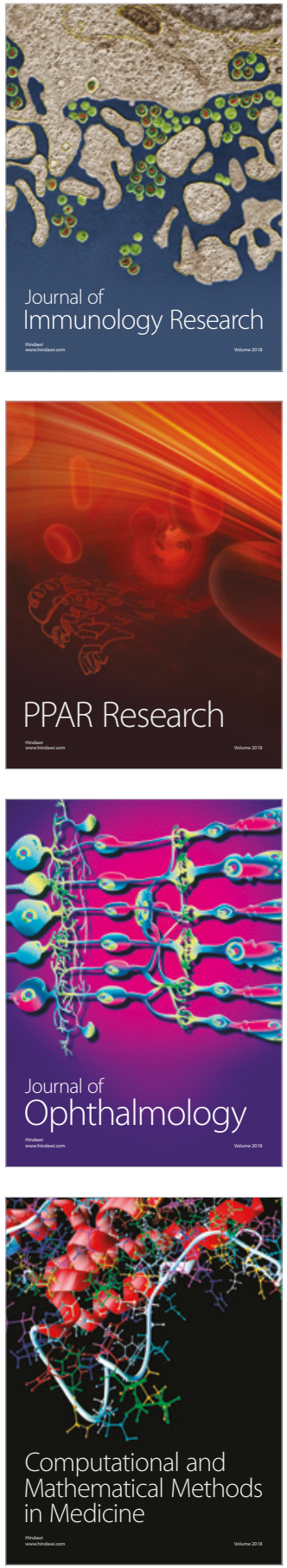

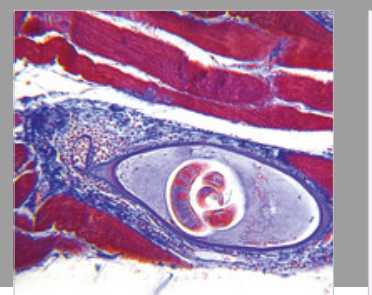

Gastroenterology Research and Practice

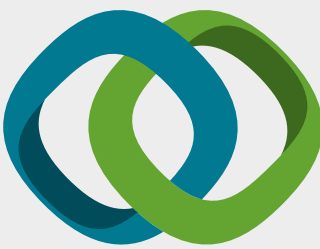

\section{Hindawi}

Submit your manuscripts at

www.hindawi.com
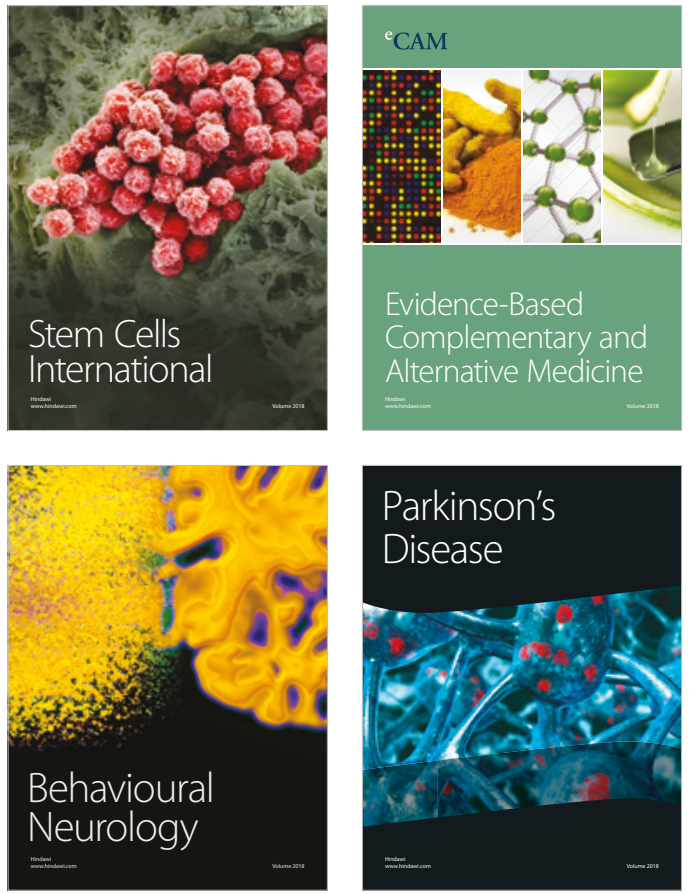

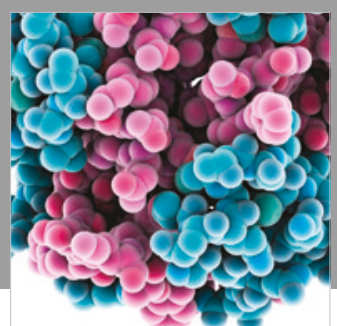

ournal of

Diabetes Research

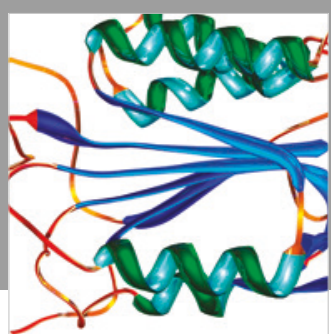

Disease Markers
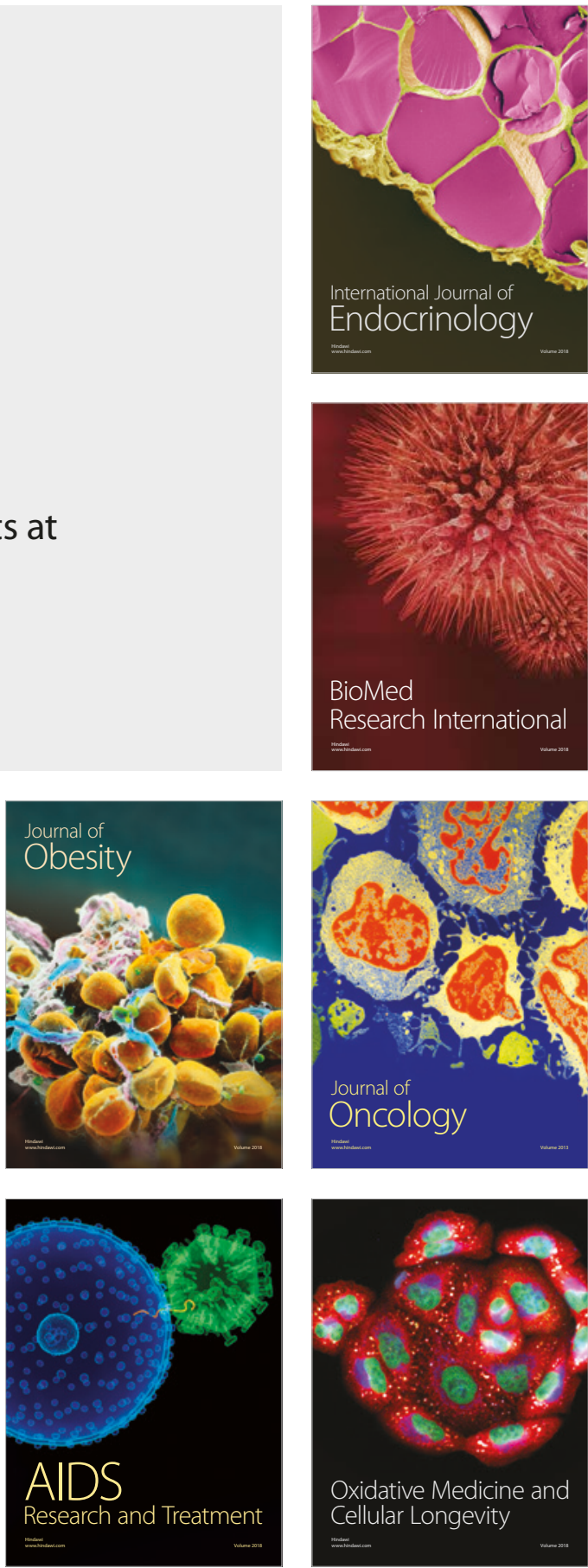